\title{
TARGET VELOCITY BASED PREDICTION IN SACCADIC VECTOR PROGRAMMING*
}

\author{
Samuel Ron, $†$ Thierry Vieville and Jacques Droulez \\ Laboratoire de Physiologie Neurosensorielle, C.N.R.S., 15 Rue de l'Ecole de Médecine, 75270 Paris, \\ Cedex 06, France
}

(Received 18 November 1986; in final revised form 6 February 1989)

\begin{abstract}
Two experiments have been designed to test whether the saccadic system takes target motion into consideration in computing saccade amplitude. In one experiment, while the subject fixated straight ahead, either a horizontal ramp-step-ramp or a horizontal step-ramp target moved from left to right. After the step, the subject had to make a saccade and follow the target. In the second set of experiments, the target, after an initial step, moved extrafoveally from up to down at fixed velocity; a tone, signaling the subject to make a saccade to the target and follow it, was delivered either after a variable delay (previewed condition) or simultaneously with the intial target step (non-previewed condition). In both experiments, eye position at saccade end was statistically different from target position $100 \mathrm{msec}$ before saccade onset only when the target slow motion was presented before the step (i.e. in horizontal ramp-step-ramp and in previewed H-step V-ramp paradigms), suggesting that target motion could be used by the saccadic system to extrapolate the future target position, only if the subject is given enough time to observe the target ramp motion before the step.
\end{abstract}

Saccade Programming Step Ramp Prediction Position error

\section{INTRODUCTION}

Rashbass (1961) introduced the step-ramp paradigm (i.e. a target which jumps to one side and immediately begins to move in the same direction at constant velocity) to show the separation of the saccadic and smooth pursuit systems. Using the same paradigm, other investigators (Carpenter, 1977; Fuchs, 1971; Robinson, 1973) concluded that the saccadic system takes the motion of the target during the ramp into account in programming the ensuing saccade. For the system to make such a prediction, it must estimate target position at the time the saccade actually occurs, approx. $200 \mathrm{msec}$ after the target jumps. More recently, Heywood and Churcher (1981), using a step-ramp paradigm (in the same directions), pointed out that the error estimated by the saccadic system might not be predictive in the strict sense: "For human subjects at least, knowledge of the task situation may lead to the adoption of response strategies that are "predictive" in the sense that

*Short communication was presented at European Brain and Behaviour Society Meeting, Tel Aviv, 26-29 May, 1986.

TOn leave from the Occupational Health and Rehabilitation Institute, at Loewenstein Hospital, Raanana, Israel. they allow the task to be performed with improved accuracy, yet do not involve prediction in the sense of extrapolation of velocity at all".

Since visual information relevant to saccadic execution seems to be unavailable within the $80-100$ msec immediately preceding saccade onset (Becker \& Jürgens, 1979; Komoda, Festinger, Phillips, Duckworth \& Young, 1973; Wheeless, Boynton \& Cohen, 1966), it was proposed that the error signal to the saccadic system was based on the target position error sampled at $100 \mathrm{msec}$ before the onset of the saccade. Thus, the data of Heywood and Churcher (1981) indicate that in a naive subject, the saccade amplitude is a linear function of target position error at $100 \mathrm{msec}$ before the saccade onset. In experienced subjects, the saccade amplitude reflects a target error approximately between the error at saccade onset and that at $100 \mathrm{msec}$ prior to saccade onset.

The lack of clear results as to whether information regarding target velocity or pursuit is actually used by the saccadic system prompted us to re-examine the question of target acquisition accuracy. Our basic hypothesis was that when a step-ramp target is pursued, the saccadic system does not have enough time to calculate the target velocity, and therefore does not 
accurately predict target location at saccade onset or end. If the saccadic reaction time is $200 \mathrm{msec}$ and if saccades are not altered $80-100 \mathrm{msec}$ before onset, then the saccadic system has only $100-120$ msec to extract information concerning velocity. Considering the delay of the visual system, this is a very short interval.

In normal situations, the subject probably observes the target motion for longer than $100-120 \mathrm{msec}$ before making an eye movement. To test whether saccadic accuracy improves if the subject observes the target movement before being instructed to make a saccade, we presented each subject with four different target motions, pooled in two experiments.

In the first experiment, in one condition, the target moved from an extrafoveal position on the horizontal axis towards the subject's central fixation then jumped in the same direction and finally moved again at the same velocity (horizontal ramp-step-ramp stimulus). In the second condition of this experiment, a simple horizontal step-ramp target motion was used. In both conditions, the subject was requested to jump on the target and to follow it immediately after the target step. Only in the first condition, did the subject observe the target velocity before he had to make a saccade.

In the second experiment, in one condition, the subject observed a target jumping from the point of his central fixation to the upper right corner and than moving extrafoveally along the vertical axis (from up to down). Following an acoustic signal (click), the subject had to make an oblique saccade to catch the target and then to follow it. The click was delivered during the target vertical ramp at some specific vertical position (previewed condition). In the second condition of this experiment, the target jumped to the right and immediately began moving down at constant velocity (non-previewed condition). The acoustic signal was given at the initial target jump.

If the saccadic system takes target movement into consideration, then saccade amplitude should correlate better with the target error at saccade onset (or end) than with the target error at $100 \mathrm{msec}$ prior to saccade onset. Results of both experiments indicate that this was achieved only when target velocity was presented to the subject before the step, i.e. in the horizontal ramp-step-ramp paradigm and in the previewed $\mathrm{H}$-step $\mathrm{V}$-ramp. In the non-previewed conditions (horizontal step-ramp and $\mathrm{H}$-step
V-ramp paradigms), eye position at saccade end was not statistically different from target position at $100 \mathrm{msec}$ before saccade onset.

\section{METHOD}

\section{Subjects and experimental setup}

Nine naive subjects, aged $20-24 \mathrm{yr}$, participated in the experiments. Three sets of experiments were conducted in one session, separated by calibration trials at the beginning and end of the session. The subjects were seated in a darkened room facing a translucent screen at $1.5 \mathrm{~m}$, with their heads fixed by a dental bite. The target was a laser beam projected on the opposite side of the translucent screen after reflection on a small mirror. The angular displacements of the mirror were driven by a galvanometer; the total inertia of this electromechanical device was negligible. Horizontal and vertical eye movements were measured in the right eye using a scleral coil technique. The overall system had a halfpower bandwidth of $200 \mathrm{~Hz}$ and a sensitivity of $0.2 \mathrm{deg}$. All experiments were run under computer control.

\section{Experimental design}

In the first condition of the first experimenthorizontal ramp-step-ramp stimulus-a total of 862 trials was performed. The subject fixated on the laser dot at the center of the screen for $2-4 \mathrm{sec}$. While the subject maintained his fixation, the target jumped $20 \mathrm{deg}$ to the left and moved at fixed velocity to the right (ramp movement). At some specific position on the horizontal axis, the target jumped to the right and simultaneously continued to move to the right at the same velocity as before the step (Fig. 1, A). The subject was asked to maintain fixation until the target jump and then to track the target as fast and as accurately as possible. The target ramp velocities $(5,15$ or $25 \mathrm{deg} / \mathrm{sec})$ and steps $(3,5$ or $10 \mathrm{deg})$ were randomly chosen for each subject. The step occurred randomly when the target was 7 or $3 \mathrm{deg}$ to the left of central fixation, at central fixation or $3 \mathrm{deg}$ to the right of central fixation.

In the second condition of the first experiment-horizontal step-ramp stimulus-a total of 360 trials was performed. The subject fixated on the laser dot at the center of the screen for 2-4 sec. The subject was requested to track the target when it jumped to the right on the horizontal axis and simultaneously moved at fixed velocity to the right (Fig. 1, B). The target 
A

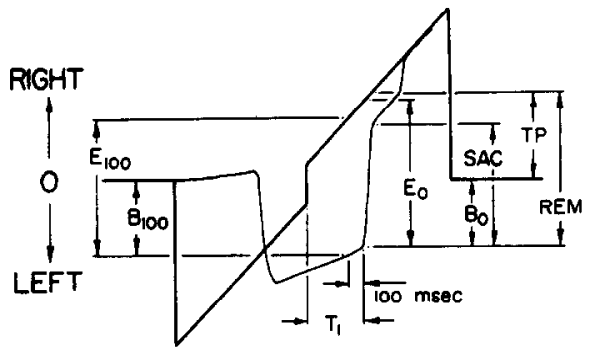

B

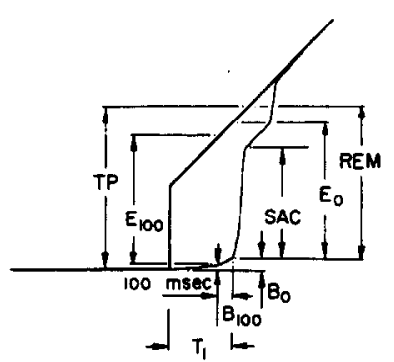

Fig. 1. Diagrammatic representation of eye movement responses and of the stimulus variables using $(A)$ the horizontal ramp-step-ramp stimulus and (B) the horizontal step-ramp stimulus. SAC, first saccade amplitude; TP, target position at saccade end in head coordinate; REM, target position at saccade end relative to eye position at saccade onset; $E_{0}$, target position in retinal coordinate at saccade onset; $\mathrm{E}_{100}$, target position in retinal coordinate at $100 \mathrm{msec}$ before saccade onset; $\mathbf{B}_{0}$, eye position in head coordinate at saccade onset; $B_{100}$, eye position in head coordinate at $100 \mathrm{msec}$ before saccade onset; $T_{1}$, latency of the first saccade.

ramp velocities and steps were as in the first experiment and were chosen randomly.

In the first condition of the second experiment-previewed H-step V-ramp stimulus-a total of 1062 trials was performed. The subject fixated on the laser dot at the center of the screen for 2-4 sec. While the subject maintained his fixation, the target jumped up $20 \mathrm{deg}$ and to the right $(5,10$ or $20 \mathrm{deg})$ and moved down $40 \mathrm{deg}$ at fixed velocity $(5,15$ or $25 \mathrm{deg} / \mathrm{sec})$ (Fig. 2, A). At some specific position on the vertical axis, a click was delivered through a loudspeaker. The subject was initially requested to remain still at his first fixation site until he heard the auditory signal, whereupon he was to track the target as fast and as accurately as possible. For each subject, the vertical ramp velocity and the horizontal step were randomly chosen. The auditory signal occurred randomly either when the target was $5 \mathrm{deg}$ above the horizontal axis, exactly on the horizontal axis, or $5 \mathrm{deg}$ below. If the oculomotor system does take target movement into consideration (for extrapolating target position), the saccade would be to location " $t_{0}$ " on the vertical axis and land accurately on target. If, on the other hand, the saccadic system samples the position error at $100 \mathrm{msec}$ before saccade onset, the saccadic jump would be to " $t_{0}-100$ " on the vertical axis and fall behind the target.

In the second condition of the second experiment-non-previewed H-step V-ramp stimulus-360 trials were performed. After 2-4 sec of central fixation, an acoustic signal was given, and the target moved to the right from the initial position and simultaneously moved down with fixed velocity (Fig. 2, B). For each subject, the vertical ramp velocities $(5,15$ or $25 \mathrm{deg} / \mathrm{sec})$ and the horizontal steps $(5,10$ or $20 \mathrm{deg})$ were randomly chosen (all combinations given to each subject). One subject did not receive the acoustic signal, but the measured variables were comparable to those of the other subjects.

A third (control) experiment-step stimulus - containing a total of 224 trials was performed. The subject fixated on the laser dot on the horizontal axis. After 2-4 sec the target jumped horizontally to a fixed displacement $(3,5,10$ or $20 \mathrm{deg}$, at random) from the initial position. To control for any possible direction preference, each experiment also contained about 10 trials in which ramp velocity and step displacement were reversed (i.e. ramp movement and step displacement to the left instead of to the right, or up target motion instead of down motion).

\section{Analysis of data}

The signals of eye and target positions were sampled on-line at a rate of $400 \mathrm{~Hz}$ and subsequently analyzed off-line. For each trial, the computer program identified the response movements for at least two consecutive saccades. The analysis was done separately for the horizontal and vertical components. For each eye movement response observed after the step (first experiment) or after the click (second experiment), the following measurments were performed (Fig. 1 and Fig. 2): latency of the first saccade $\left(T_{1}\right)$ and latency of the second (correcting) saccade $\left(\mathrm{T}_{2}\right)$; first saccade amplitude (SAC); eye position in head coordinate at saccade onset $\left(B_{0}\right)$ and at $100 \mathrm{msec}$ before saccade onset $\left(B_{100}\right)$; target position in retinal coordinate at saccade onset $\left(E_{0}\right)$ and at $100 \mathrm{msec}$ before saccade onset $\left(\mathrm{E}_{100}\right)$; target position in head coordinate at saccade end (TP); target position at saccade end relative to eye position at saccade onset (relative expected movement, REM). 
A

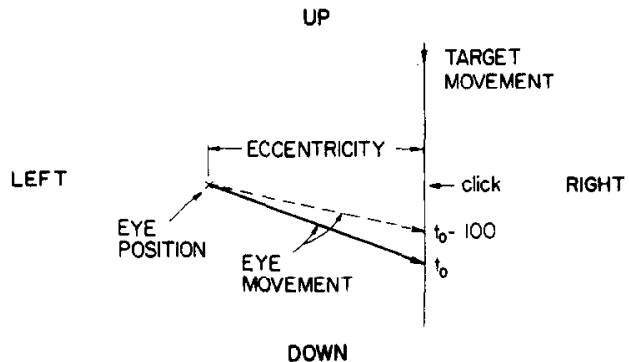

$B$
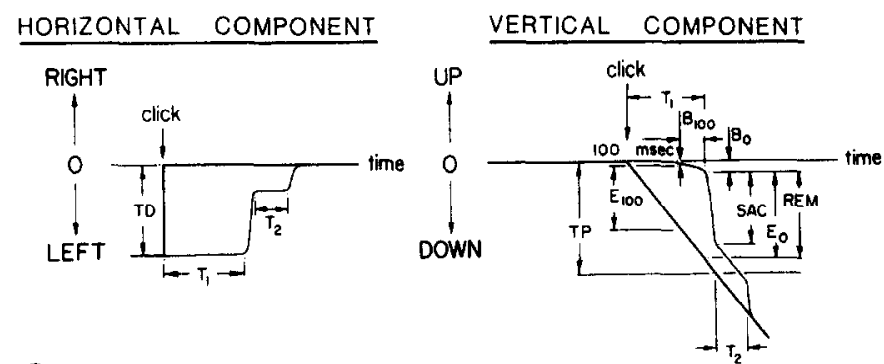

C
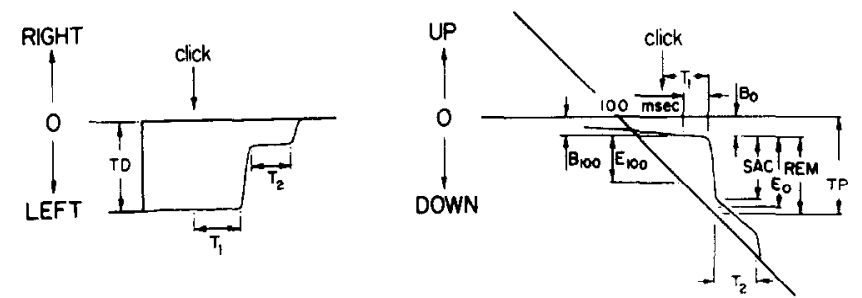

Fig. 2. (A) Two-dimensional diagram showing the previewed H-step V-ramp paradigm. Initially, the eye fixates at a target which, after a few seconds, jumps to the right and up and moves down at a fixed velocity. At some predetermined site, an auditory signal is delivered, followed by the saccadic response. The two vectors representing eye movements are two possible saccadic jumps, depending on whether the saccadic amplitude is based on position error at $100 \mathrm{msec}$ before saccade onset (interrupted line) or on position error at saccade onset (solid line). (B) and (C) Diagrammatic presentation of the stimulus and the eye movements in the two paradigms of the second experiment, (B) non-previewed H-step V-ramp, and (C) previewed H-step V-ramp. SAC, first saccade amplitude; TP, target position in head coordinate at saccade end; REM, target position at saccade end relative to eye position at saccade onset; $\mathrm{E}_{0}$, target position in retinal coordinate at saccade onset; $E_{100}$, target position in retinal coordinate at $100 \mathrm{msec}$ before saccade onset; $\mathbf{B}_{0}$, eye position in head coordinate at saccade onset; $\mathbf{B}_{100}$, eye position in head coordinate at $100 \mathrm{msec}$ before saccade onset; TD, horizontal target displacement; $T_{1}$, latency of the first saccade using ramp-step-ramp stimuli; $T_{2}$, latency of the correcting saccade.

Except for latencies $\left(T_{1}\right.$ and $\left.T_{2}\right)$, which were measured on both horizontal and vertical axes, all the above parameters were measured on the target ramp axis, i.e. the horizontal axis in the first experiment and the vertical axis in the second experiment.

Eye movements that either preceded or lagged by less than $100 \mathrm{msec}$ behind the target jump or the auditory signal were excluded. In the rampstep-ramp stimulus trials, eye movements made prior to the step of velocity of more than half the target velocity were excluded from the analysis. Also excluded were eye movements that were driven, prior to the auditory signal in the second experiment, to a fixation point outside a $10 \mathrm{deg}$ range from the initial fixation. The latter were primarily the result of lack of attention by the subject.

\section{Statistical analysis}

The statistical analysis was done to determine whether the saccadic parameters were based on the target position error at $100 \mathrm{msec}$ before saccade onset or on the error at the onset or the end of the saccade, which would imply the use of target velocity information. In the previewed paradigms, some of the eye movements prior to the step (in the horizontal ramp-step-ramp 
experiment) or the auditory signal (in the previewed $\mathrm{H}$-step $\mathrm{V}$-ramp experiment) were driven by target movement. It was therefore necessary to calculate the variables once from the physical axis (head coordinate) and again with respect to saccade onset (retinal coordinate). For each of the three target velocities, the difference between the saccade amplitude and each independent variable was tested using a multivariable test (paired Hotelling $\mathrm{T}^{2}$ test) and a univariate test (multiple paired $t$-test) (Tatsuoka, 1971). Also computed were the correlations, collapsed across target motion velocities, of the eye position at saccade end with the independent variables.

\section{RESULTS}

\section{Presaccadic eye movements}

When the previewed ramp velocity stimuli were used, the eyes tended to make extrafoveal pursuit movements before the step (Logothetis, Fries \& Popel, 1985). The velocity of this motion was either low and uncorrelated to the target ramp velocity (Fig. 3, A) or approximately equal to the target ramp velocity (Fig. 3, B). In the former case, the saccades before the step could be in either direction, whereas in the latter case, they were all in one direction and, together with the slow movement, resembled nystagmus.

The extrafoveal pursuit movement was always in the direction of the target motion, and
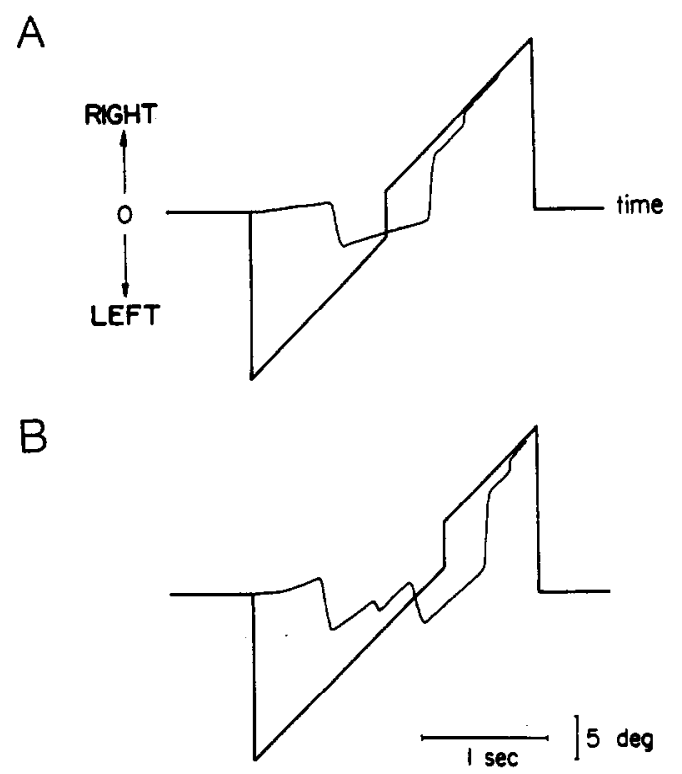

Fig. 3. Two examples of eye movement tracing in horizontal ramp-step-ramp stimulus trials. in the control sessions, when target motion was reversed (i.e. from left to right or from up to down), the direction of eye movements was also reversed. In Fig. 3, B in which the velocity of pursuit was close to target velocity, eye fixation was 3-6 deg from target position, probably outside the region of the "pursuit zone" (Eckmiller, 1981; Logothetis et al., 1985), believed by some investigators to be as large as the entire macula (Eckmiller, 1981). Early in the experimental session, extrafoveal pursuit movements were of low velocity $(<5 \mathrm{deg} / \mathrm{sec})$, but the velocity increased later in the session. We believe that, at least in part, extrafoveal pursuit was influenced by the alertness and attention of the subject.

Similar results were observed on the vertical axis in the previewed H-step V-ramp experiment. The vertical drift velocity was in the range of $1.0-8.0 \mathrm{deg} / \mathrm{sec}$ and was only weakly correlated to target velocity $(r=0.28)$. Since in this condition, the target was always presented extrafoveally $(5,10$ or 20 deg from the central fixation point), we also related the vertical drifts to pursuit movements from regions outside the "pursuit zone" (Fig. 4, C). The majority of vertical drifts appeared when target motion on the vertical axis was displaced from the visual fixation by $5 \mathrm{deg}$ or less (in $17 \%$ of the responses); vertical drifts occurred less frequently for larger displacements (in $8 \%$ for $10 \mathrm{deg}$ and in $3 \%$ for $20 \mathrm{deg}$ ). The direction of the saccade vertical component (i.e. up or down) depended on the position of the target when the auditory signal was given and on the accumulated drift at the last position at which that error was sampled by the saccadic system before saccade onset. In a few cases $(<3 \%)$, the vertical drift developed into a small nystagmus-like trajectory (Fig. 4, D).

In the previewed $\mathrm{H}$-step V-ramp experiment, small horizontal drifts preceding the auditory signal were also observed. The horizontal drifts measured approx. $0.5-1.0 \mathrm{deg} / \mathrm{sec}$ and were independent of target displacement $(t=1.40$, $P>0.1)$ or target velocity in the vertical direction $(t=1.18, P>0.1)$. When target displacement was to the left, the horizontal drift was either to the left or to the right.

\section{Saccade latency}

In the horizontal ramp-step-ramp stimulus trials, the saccade latency $\left(T_{1}\right)$ decreased for higher ramp velocities $(P<0.01, t=9.7)$ and was independent of the size of the target jump $(P>0.1, t=1.1)$ (Table 1). Single saccades 


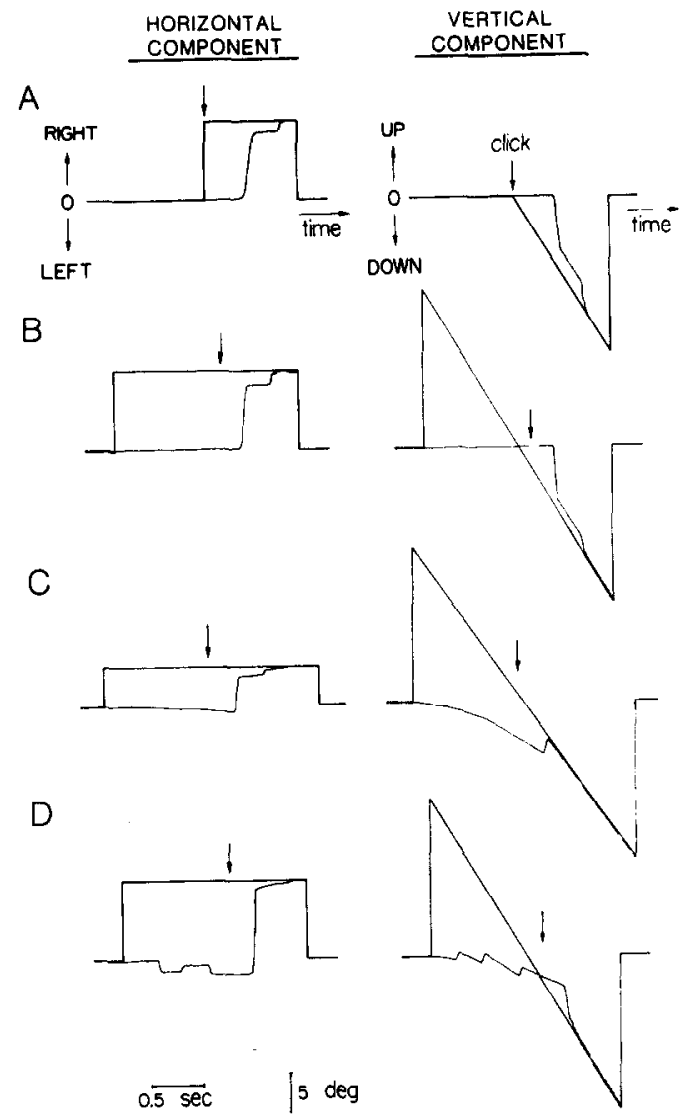

Fig. 4. (A) An example of eye movement tracing with the non-previewed $\mathrm{H}$-step V-ramp paradigm. (B-D) Three examples of eye movement tracing with the previewed H-step V-ramp stimulus. Left panel, horizontal components; right panel, vertical components. The arrow indicates the time at which the auditory signal (click) was delivered.

were found in $56 \%$ of the trials, and two saccades in $42 \%$. The occurrence of two saccades depended on target step size and on the movements of the eyes before the step. When a second saccade was present, the mean inter-

Table 1. Mean latency of the first saccade for each of the three stimulus velocities, and for each of the four paradigms: horizontal ramp-step-ramp, horizontal step-ramp, previewed $\mathrm{H}$-step V-ramp and non-previewed H-step V-ramp. Standard deviations are indicated in parenthesis

\begin{tabular}{lccc}
\hline & \multicolumn{3}{c}{ Stimulus velocity (deg/sec) } \\
\cline { 2 - 4 } Experimental conditions & 5 & 15 & 25 \\
\hline $\begin{array}{l}\text { First experiment } \\
\text { Horizontal ramp- } \\
\quad \text { step-ramp }\end{array}$ & $287(63)$ & $263(52)$ & $238(54)$ \\
$\quad \begin{array}{l}\text { Horizontal step-ramp } \\
\text { Second experiment } \\
\text { Previewed H-step } \\
\quad \text { V-ramp }\end{array}$ & $209(48)$ & $223(53)$ & $205(50)$ \\
$\quad$ Non-previewed H-step & $313(66)$ & $335(68)$ & $315(75)$ \\
$\quad$ V-ramp & $267(53)$ & $335(62)$ & $373(70)$ \\
\hline
\end{tabular}

saccade latency $\left(\mathrm{T}_{2}\right)$ was $214 \mathrm{msec}$ and was independent of ramp velocity.

Mean saccade latency in the horizontal stepramp stimulus trials was $212 \mathrm{msec}(\mathrm{SD}=51)$ and was not dependent on ramp velocity $(P>0.1, t=0.9)$. These results are in general agreement with those of Heywood and Churcher (1981) and contrary to the suggestion by Robinson (1965) that saccade latency is dependent on stimulus ramp velocity. Single saccades were found in $66 \%$ of the trials. The mean latency of the second saccade was $198 \mathrm{msec}(\mathrm{SD}=42)$, which was similar to that found in other studies (Heywood \& Churcher, 1981; Robinson, 1965).

Similarly, when the previewed H-step V-ramp stimulus was presented (second experiment), the latency of the first saccade $\left(T_{1}\right)$ was not dependent on target velocity $(t=0.96, P>0.1)$. The saccadic delay ( $m=296-325 \mathrm{msec}$, Table 1$)$ was within the range recorded in response to an auditory signal, when the subject was asked to jump to a target's spatial location (250-350 msec, depending on target position) (Zambarbieri, Schmid, Magenes \& Prablanc, 1982). This latency was not dependent on the horizontal step size or on the location of the target on the vertical axis at the time of the signal $(t=1.03, P>0.1$ and $t=1.36, P>0.1$, respectively). Correcting saccades were found in $57 \%$ of the trials; their mean latency $\left(\mathrm{T}_{2}\right)$ was $m=225 \mathrm{msec}(\mathrm{SD}=64)$ and was independent of the target velocity $(t=1.28, P>0.1)$.

The majority of the saccadic correction components were generated together, resulting in an oblique movement (Fig. 4, B). When separate movements were made to correct the horizontal and vertical errors, the vertical correcting saccade usually preceded the horizontal. Some of these vertical correcting saccades had a very short latency $(m=130-160 \mathrm{msec})$. For 10 or 20 deg horizontal steps, $96 \%$ of the correcting saccades were oblique.

In response to an $\mathrm{H}$-step $\mathrm{V}$-ramp stimulus (non-previewed condition, second experiment), the latency of the first saccade $\left(T_{1}\right)$ increased with target velocity (Table 1). This latency was longer than that to a horizontal step-ramp stimulus (Heywood \& Churcher, 1981). Correcting saccades were found in $66 \%$ of the trials; their mean latency $\left(\mathrm{T}_{2}\right)$ was $m=233 \mathrm{msec}$ $(\mathrm{SD}=57)$ and was independent of the stimulus velocity $(t=1.42, P>0.1)$. For 10 or $20 \mathrm{deg}$ horizontal steps, $96 \%$ of the correcting saccades were oblique (Fig. 4, A). 


\section{Saccade amplitude}

To test whether target motion before the step influences the first saccade, we plotted eye position at saccade end $\left(B_{0}+S A C\right)$ versus target position at $100 \mathrm{msec}$ saccade onset $\left(\mathrm{B}_{100}+\mathrm{E}_{100}\right)$, and versus target position at saccade end (TP). As mentioned above, these parameters were analyzed on the axis along which the target slow motion occurred, i.e. the horizontal axis in the first experiment and the vertical axis in the second experiment. In the latter case, the horizontal component of the first saccade was usually hypometric and was followed by a small correcting saccade.

In the previewed horizontal ramp-step-ramp paradigm, linear regressions of both plots had comparable slopes (0.9-0.92), but the intercept of the former regression in Fig. 5, A indicated that there was a larger error in matching target position at $100 \mathrm{msec}$ before saccade end than in matching target position at saccade end

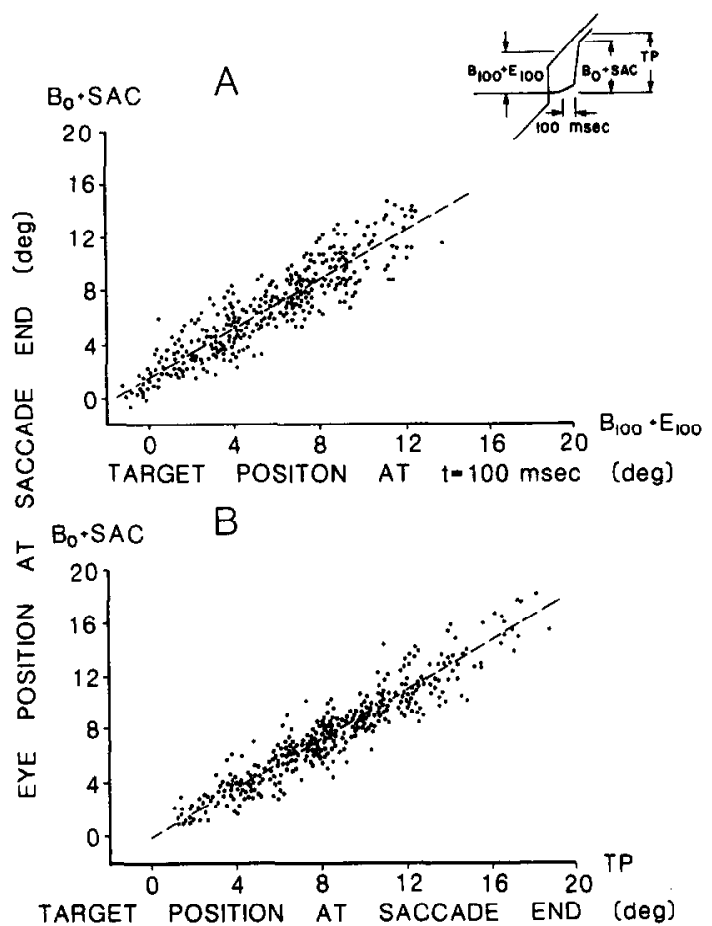

Fig. 5. The individually measured data when the horizontal ramp-step-ramp stimulus was used. Step size was $5 \mathrm{deg}$. The step occurred when the target was at $3 \mathrm{deg}$ to the left of central fixation. (A) Eye position at saccade end $\left(B_{0}+S A C\right)$ vs target position at $100 \mathrm{msec}$ before saccade onset $\left(B_{100}+E_{100}\right)$ and the fitted linear regression $B_{0}+S A C=0.9\left(B_{100}+E_{100}\right)+1.2(n=306)$. (B) Eye position at saccade end $\left(B_{0}+S A C\right)$ vs target position at saccade end (TP) and the fitted linear regression $\mathrm{B}_{0}+\mathrm{SAC}=0.92$ (TP) $-0.22(n=320)$. Negative values indicate that the measured values were below the horizontal axis. Inset, the measured variables.
(1.2 vs -0.22 ). For horizontal step-ramp stimuli, the computed linear regression slope in Fig. 6, A was closer to 1.0 (about the same value as in Fig. 5, A), with again a higher intercept than in Fig. 6, B (1.75 vs 0.83 ).

These data indicated that, on average, small saccades ended closer to the actual target position than did large saccades, elicited by large target errors, which ended in the vicinity of the position reached by the target $100 \mathrm{msec}$ before the saccade onset.

Similarly, for the previewed (Fig. 7) and non-previewed (Fig. 8) H-step V-ramp paradigms of the second experiment, we plotted vertical eye position after the first saccade $\left(B_{0}+S A C\right)$ vs vertical target position at 100 msec before saccade onset $\left(B_{100}+E_{100}\right)$, and vs vertical target position at saccade end (TP). In the previewed condition, the slopes of the two linear regressions were about the same ( 0.873 vs 0.83 ), but the intercept of the former linear regression (Fig. 7, A) was much larger than the latter (3.9 vs 1.13) (Fig. 7, B). This indicated that eye position was more nearly

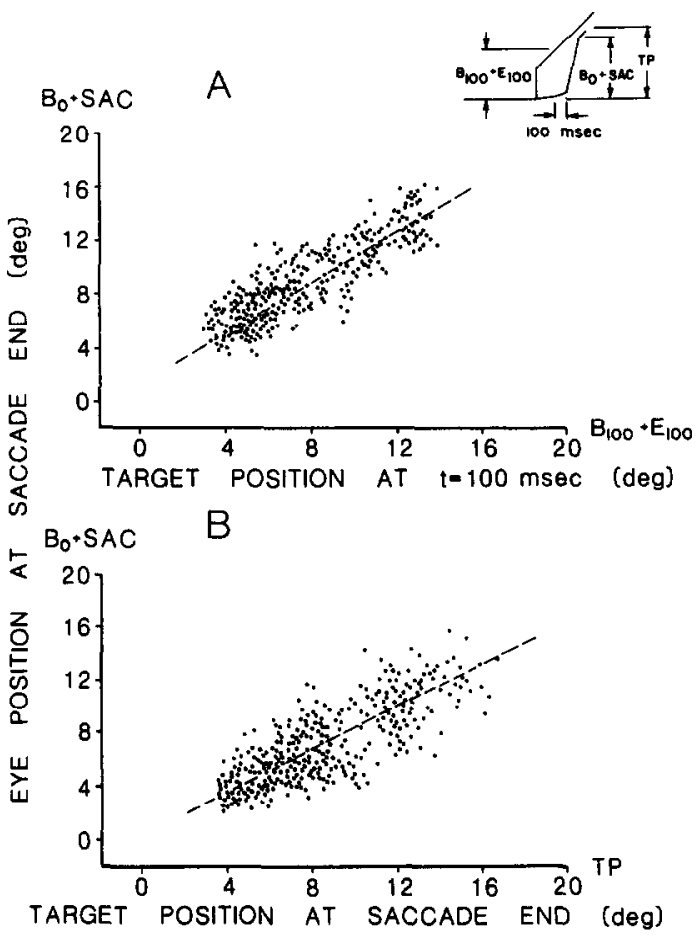

Fig. 6. The individually measured data when the horizontal step-ramp stimulus was used. Step size was $5 \mathrm{deg}$. (A) Eye position at saccade end $\left(B_{0}+S A C\right)$ vs target position at $100 \mathrm{msec}$ before saccade onset $\left(B_{100}+E_{100}\right)$ and the fitted linear regression $B_{0}+S A C=0.94 \quad\left(B_{100}+E_{100}\right)+1.75$ $(n=312)$. (B) Eye position at saccade end $\left(\mathrm{B}_{0}+\mathrm{SAC}\right)$ vs target position at saccade end (TP) and the fitted linear regression $\mathrm{B}_{0}+\mathrm{SAC}=0.8(\mathrm{TP})+0.83(n=311)$. Inset, the measured variables. 

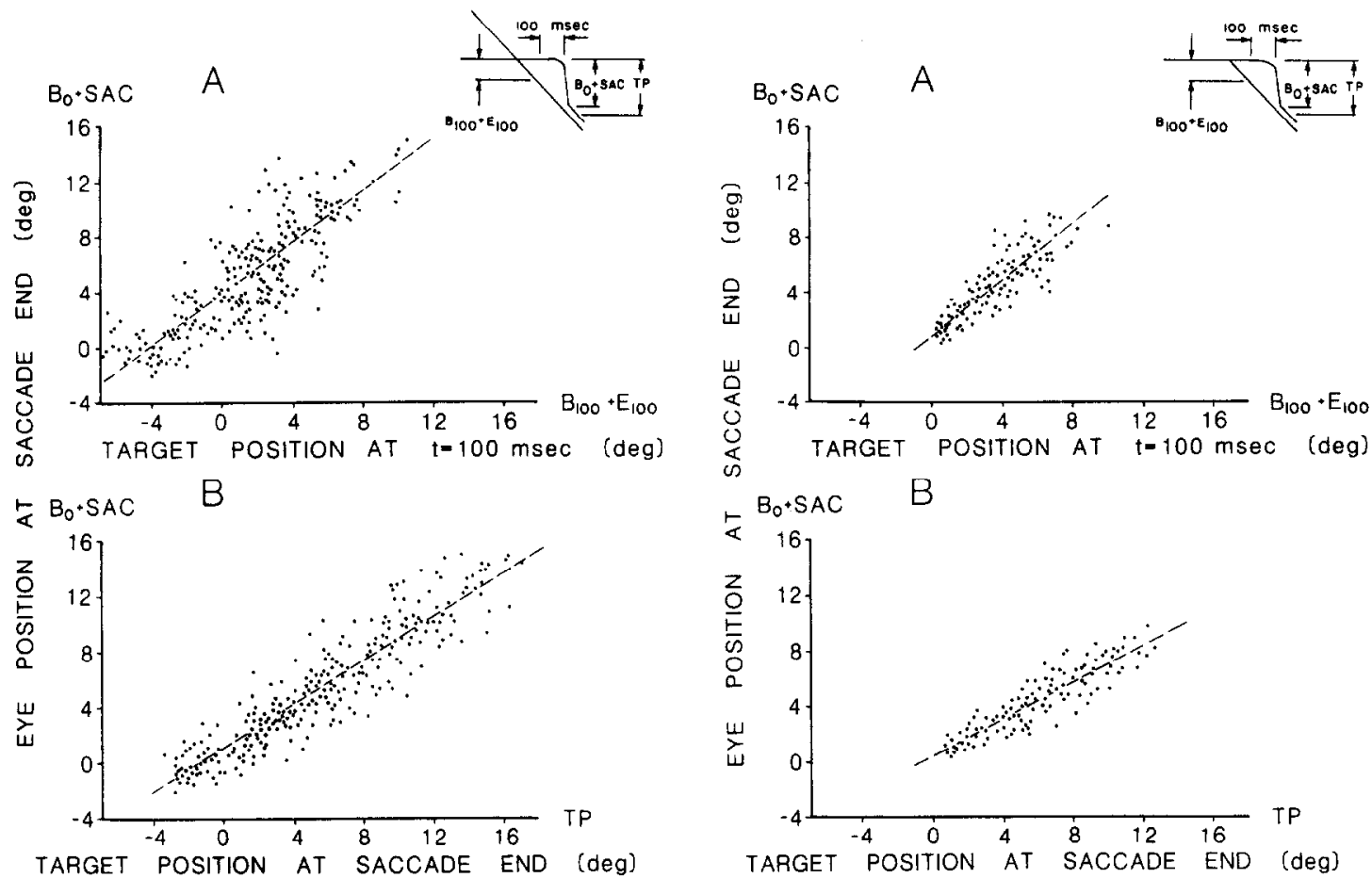

Fig. 7. The individually measured data of the vertical components when stimulus was the previewed $\mathrm{H}$-step Vramp. The horizontal step was $10 \mathrm{deg}$, and the auditory signal was given when the descending target crossed the horizontal axis. (A) Eye position at saccade end $\left(B_{0}+S A C\right)$ vs target position 100 msec before saccade onset $\left(B_{100}+\right.$ $\left.E_{(w 0)}\right)$ and the fitted linear regression $B_{0}+S A C=0.88$ $\left(B_{100}+E_{100}\right)+3.9(n=251)$. (B) Eye position at saccade end $\left(\mathrm{B}_{0}+\mathrm{SAC}\right)$ vs target position at saccade end (TP) and the fitted linear regression $B_{0}+\mathrm{SAC}=0.81 \quad(\mathrm{TP})+1.13$ $(n=252)$. Inset, the measured variables.

equal to target position at saccade end than at $t=100 \mathrm{msec}$. In contrast, when the target vertical velocity was not previewed, the eye position at saccade end was more nearly equal to target position at $100 \mathrm{msec}$ before saccade onset for all amplitudes: the slope of the former linear regression was higher $(0.93)$ (Fig. 8, A) than the slope of the latter $(0.67)$ (Fig. 8, B), whereas the intercepts were about the same ( 0.8 vs 0.5$)$.

To test the change in these correlations as a result of different target velocities, we computed the following four variables: target position at saccade end (TP), at saccade onset $\left(B_{0}+E_{0}\right)$ and at $100 \mathrm{msec}$ before saccade onset $\left(B_{100}+E_{100}\right)$, and eye position at saccade end $\left(\mathrm{B}_{0}+\mathrm{SAC}\right)$. We plotted the mean and standard deviations of these four variables vs the target velocity in the two experiments (Figs 9 and 10).

In the first experiment, for horizontal rampstep-ramp stimuli (Fig. 9, A), eye position at saccade end was statistically different from target position at $100 \mathrm{msec}$ before saccade onset

Fig. 8. The individually measured data of the vertical components when stimulus was the non-previewed H-step V-ramp and horizontal step was 10 deg. (A) Eye position at saccade end $\left(B_{0}+S A C\right)$ vs target position at $100 \mathrm{msec}$ before saccade onset $\left(B_{100}+E_{100}\right)$ and the fitted linear regression $\mathrm{B}_{0}+\mathrm{SAC}=0.93\left(\mathrm{~B}_{100}+\mathrm{E}_{100}\right)+0.8 \quad(n=113)$. (B) Eye position at saccade end $\left(B_{0}+S A C\right)$ vs target position at saccade end (TP) and the fitted linear regression $\mathrm{B}_{0}+\mathrm{SAC}=0.67(\mathrm{TP})+0.5(n=108)$. Inset, the measured variables.

$(F(3,2)=76.4, P<0.05)$. The $t$-test for these variables was significant at stimulus velocities of $15 \mathrm{deg} / \mathrm{sec}(t=2.55, P<0.05)$ and $25 \mathrm{deg} / \mathrm{sec}$ $(t=5.66, \quad P<0.01)$. When horizontal stepramp stimuli were used (Fig. 9, B), eye position at saccade end was statistically different from target position at saccade end $(F(3,2)=32.8$, $P<0.05)$. The $t$-test was significant at stimulus velocities of $15 \mathrm{deg} / \mathrm{sec}(t=2.48, P<0.05)$ and $25 \mathrm{deg} / \mathrm{sec}(t=5.84, P<0.01)$.

For the second experiment, the mean and standard deviations of the same four variables are plotted in Fig. 10. In the previewed H-step V-ramp stimulus trials (Fig. 10, A), eye position at saccade end $\left(B_{0}+\mathrm{SAC}\right)$ was statistically different from target position at $100 \mathrm{msec}$ before saccade onset $\left(\mathrm{B}_{100}+\mathrm{E}_{100}\right) \quad(F(3,3)=88.6$, $P<0.05)$ at stimulus velocities of $15 \mathrm{deg} / \mathrm{sec}$ $(t=2.65, P<0.05)$ and $25 \mathrm{deg} / \mathrm{sec}(t=4.63$, $P<0.01)$. The difference between $\left(B_{0}+\right.$ SAC) and $\left(B_{100}+E_{100}\right)$ progressively increased with increased velocity. At least for higher velocities, 

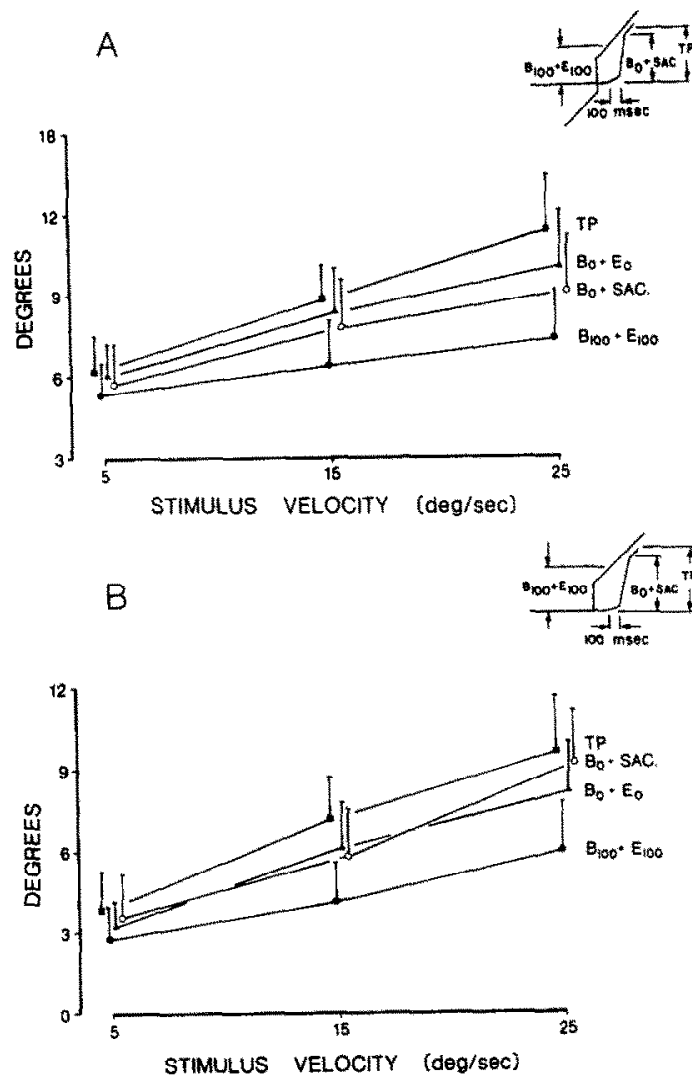

Fig. 9. Mean and standard deviations of the four measured variables vs stimulus velocity when the stimulus presented was (A) the horizontal ramp-step-ramp, or (B) the horizontal step-ramp. Step size was $S \mathrm{deg}$, and in the former paradigm, the step occurred when the target was $3 \mathrm{deg}$ to the left of central fixation. The measured variables were eye position at saccade end $\left(B_{0}+S A C\right)$, target position at saccade onset $\left(B_{0}+E_{0}\right)$, target position at $100 \mathrm{msec}$ before saccade onset $\left(B_{100}+E_{100}\right)$ and the distance the target had moved by the end of the saccade (TP). Inset, diagrammatic presentation of the paradigms and the measured variables.

these data suggested that previewing target motion improved target acquisition accuracy.

In contrast, for the non-previewed H-step V-ramp paradigm (Fig. 10, B), eye position at saccade end $\left(B_{0}+\right.$ SAC) was statistically different from target position at saccade end (TP) $(F(3,3)=62.3, P<0.05)$ at stimulus velocities of $15 \mathrm{deg} / \mathrm{sec}(t=2.45, P<0.05)$ and $25 \mathrm{deg} / \mathrm{sec}$ $(t=3.92, P<0.01)$. Eye position at saccade end was not statistically different from target position at saccade onset or at $100 \mathrm{msec}$ before onset. These data did not support the contention that velocity information is incorporated in planning saccade amplitude when non-previewed stimuli are used.

The correlation of eye position at saccade end with the independent variables collapsed across

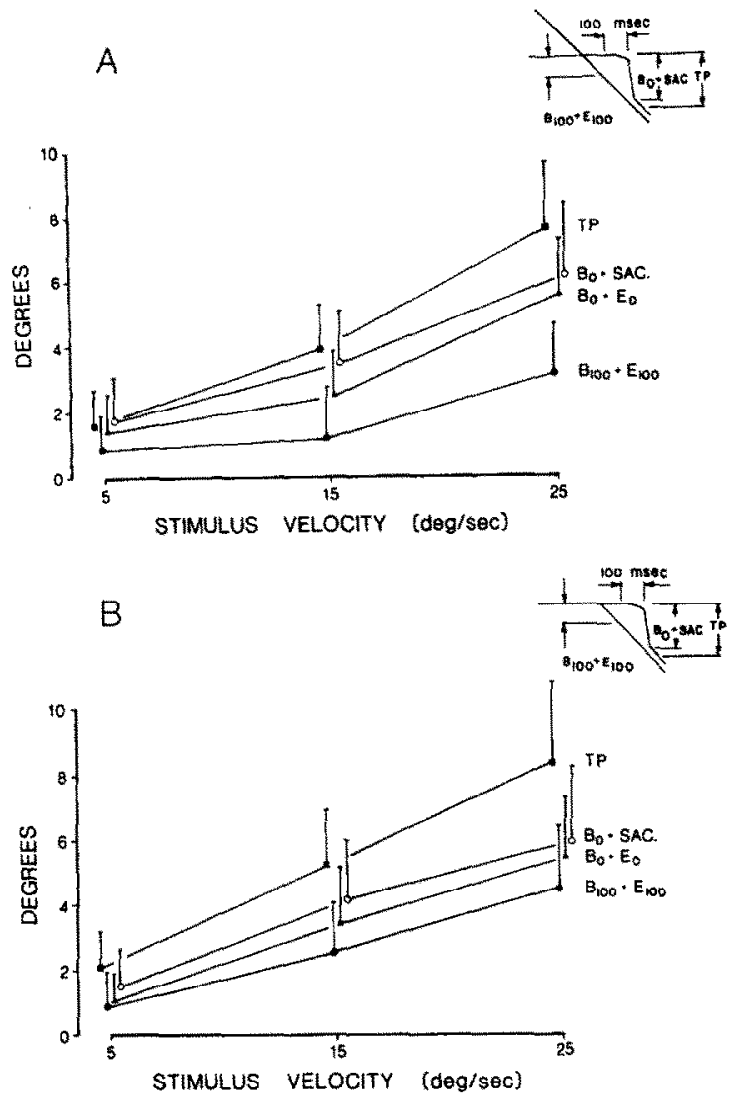

Fig. 10. Mean and standard deviations of the four measured variables vs stimulus velocity when the stimulus presented was (A) the previewed $H$-step V-ramp, or (B) the nonpreviewed H-step V-ramp. Horizontal step size was $10 \mathrm{deg}$, and in the former paradigm, the auditory signal (click) was given when the descending target crossed the horizontal axis. The measured variables were eye position at saccade end $\left(B_{0}+S A C\right)$, target position at saccade onset $\left(B_{0}+E_{0}\right)$, target position at 100 msec before saccade onset $\left(B_{100}+E_{100}\right)$ and the distance the target had moved by the end of the saccade (TP). Inset, diagrammatic presentation of the paradigms and the measured variables.

target velocities was computed for the four experimental conditions (Table 2). In the first experiment (horizontal ramp-step-ramp or stepramp stimuli), target position error at $100 \mathrm{msec}$ before saccade onset $\left(B_{100}+E_{100}\right)$ was not statistically different from either of the other two variables $(P>0.05)$. For horizontal ramp-stepramp stimuli, there was a higher correlation between eye position and target position at saccade end than with either of the other independent variables. In contrast, $\left(B_{100}+E_{100}\right)$ was statistically different from TP in both previewed and non-previewed conditoins of the second experiment (H-step V-ramp).

The statistical significance of any of the correlations did not change for different step amplitudes. Since the drift of eye movements 
Table 2. For each of the four paradigms, correlation between eye position at saccade end and the independent variables: target position at $100 \mathrm{msec}$ before saccade onset $\left(B_{100}+E_{100}\right)$, at saccade onset $\left(B_{0}+E_{0}\right)$ and at saccade end (TP). In the first two paradigms, step size was $5 \mathrm{deg}$, and in the ramp-step-ramp stimulus trials, the step was given when the target was 3 deg to the left of central fixation. In the last two paradigms, horizontal step was $10 \mathrm{deg}$, and for the previewed H-step V-ramp stimulus, the auditory signal (click) was given when the descending target crossed the horizontal axis

\begin{tabular}{lccc}
\hline & \multicolumn{3}{c}{ Target position error } \\
\cline { 2 - 4 } Experimental conditions & $\mathrm{B}_{100}+\mathrm{E}_{100}$ & $\mathrm{~B}_{0}+\mathrm{E}_{0}$ & $\mathrm{TP}$ \\
\hline $\begin{array}{l}\text { First experiment } \\
\text { Horizontal ramp- } \\
\quad \text { step-ramp }\end{array}$ & 0.902 & 0.936 & 0.941 \\
$\begin{array}{l}\text { Horizontal step-ramp } \\
\text { Second experiment } \\
\text { Previewed H-step } \\
\quad \text { V-ramp }\end{array}$ & 0.897 & 0.906 & 0.887 \\
$\begin{array}{l}\text { Non-previewed H-step } \\
\text { V-ramp }\end{array}$ & 0.873 & 0.827 & $0.923^{*}$ \\
\hline
\end{tabular}

*Significantly different from $\left(\mathrm{B}_{100}+\mathrm{E}_{100}\right)$ at $P<0.05$, two-tailed.

preceding the first saccade can alter the statistical outcome, all analyses were recomputed when variables were calculated in retinal coordinates (REM, $E_{0}, \mathrm{SAC}$ and $\mathrm{E}_{100}$ ). The only variable that changed by an amount other than $B_{0}$ was $\mathrm{E}_{100}$. As expected, the statistical significances were the same in all cases, since retinal slip velocity varied with drift.

\section{DISCUSSION}

Saccade amplitude cannot be modified during the $80-100$ msec immediately preceding saccade onset (Becker \& Jürgens, 1979; Komoda et al., 1973; Wheeless et al., 1966), or $50 \mathrm{msec}$ before the saccade movement, if the ramp velocity varies (Barmark, 1970). Hence, the available time for the subject to extract ramp velocity information is shorter than the saccadic response latency. In the present study, we questioned whether target acquisition accuracy improves if subjects observe target motion before they are requested to follow it. We compared responses to horizontal step-ramp stimuli or H-step V-ramp stimuli with those to either horizontal ramp-step-ramp (first experiment) or previewed H-step V-ramp stimuli (second experiment), when the subject could "see" the motion before the step.

Our study suggests that under previewed target motion conditions (horizontal ramp-stepramp or previewed H-step V-ramp stimuli), target velocity is taken into consideration in computing saccade amplitude. Apparently, if the subject is given enough time to observe the target ramp motion, the saccadic system can approximate target position at saccade end more accurately. Statistically, for higher target velocities, eye position at saccade end was not based on the target position error at $100 \mathrm{msec}$ before saccade onset, provided that target velocity was previewed. These findings are in agreement with those of Robinson (1973), who suggested that the saccade generator may use retinal velocity errors to extrapolate future target position.

In a recent study, McKenzie and Lisberger (1986) presented a flash during smooth pursuit of another target, so that there was a smooth change in eye position after the flash. They demonstrated that the saccade generator does not normally use non-visual feedback about smooth changes in eye position; the saccade amplitude and direction were appropriate to the target position error determined by the eye position at the time of the flash. Thus, the saccades were not corrected for intervening smooth changes in eye position. In our study, on the other hand, when the eyes made a saccade to a previewed extrafoveal moving target, the estimation of target position at saccade end was improved (Fig. 10, A); i.e. the saccades were corrected if retinal slip velocity was available for long enough periods of time. Note that in the paradigm of McKenzie and Lisberger (1986), since the target was flashed, its retinal velocity was not available.

For horizontal step-ramp or H-step V-ramp stimuli, however, eye position at saccade end was not statistically different from target position error at $100 \mathrm{msec}$ before saccade onset or at saccade end. Thus, saccade amplitude could be based on target position error sampled $100 \mathrm{msec}$ before saccade onset or target position error at the time of the saccade. Using either of these paradigms, it cannot be concluded whether or not the saccadic system extracts velocity information.

The strongest evidence that the saccadic system uses velocity information in a step-ramp paradigm was provided by Rashbass (1961). In some of his experiments, the target moved in an opposite direction to the step. Under certain combinations of step amplitude and ramp velocity, no saccade at all occurred. This "cancellation" would be possible only if movement information were taken into account. Heywood 
and Churcher (1981) could only partially confirm this finding. They argued that "cancellation is not prima facie evidence for prediction, unless the magnitude of the error at the last available sample is sufficient, given the task, to elicit the saccade". Using step-ramp stimuli (non-previewed condition), our results are in general agreement with the latter study in which in one (naive) subject, saccade amplitude was based of the target position error at $100 \mathrm{msec}$ before the saccade onset; in two additional (experienced) subjects, the saccade amplitude reflected a target position error approximately between that at saccade onset and that at $100 \mathrm{msec}$ prior to the saccade.

When presented with horizontal ramp-stepramp or previewed $\mathrm{H}$-step V-ramp stimuli, subjects tried to suppress the extrafoveal target motion by using slow eye movements in the direction of the target motion. If the target motion is outside the "pursuit zone" (Eckmiller, 1981; Logothetis et al., 1985), the subjects are only partially successful, since the eye movements are of much lower velocity than the target motion. Similar presaccadic pursuit movements were also observed in a previous study using step-ramp stimuli (Logothetis et al., 1985). These authors suggested that the function of presaccadic pursuit might be prediction of the ramp movement. In the present work, however, the presaccadic slow movements, as observed in the horizontal step-ramp or H-step V-ramp paradigms, did not improve target acquisition accuracy. This suggests that, at least in these cases, the presaccadic eye movement does not have a predictive function.

When slow drift occurred on the horizontal axis in the previewed H-step V-ramp paradigm, it was either toward the eccentrically moving target or in the opposite direction. This finding does not support the data of Wyatt and Pola (1981), who reported slow eye movement towards an extrafoveal target, with eye velocity increasing for larger target eccentricity. One possible explanation for the different results is that, in the latter study, the slow movement was toward a fixed target.

The latency of the primary saccade in the non-previewed horizontal step-ramp paradigm, as well as in the previewed H-step V-ramp paradigm, was not dependent on target velocity. These results, in agreement with those of Heywood and Churcher (1981), might however be due to different oculomotor strategies. In the horizontal step-ramp paradigm, the subject was responding to a pure visual stimulation (the step) with a short delay $(200-220 \mathrm{msec})$, whereas in the previewed $\mathrm{H}$-step V-ramp paradigm, the subject was responding to an auditory signal which induced a longer latency (Zambarbieri et al., 1982); moreover, he could take into account the previewed target velocity to improve saccade accuracy. A similar effect could explain why the latency is slightly reduced at high target velocity in the horizontal rampstep-ramp paradigm. On the other hand, in the non-previewed H-step V-ramp paradigm, the subject was faced with the task of suddenly estimating the velocity of target motion as well as its eccentricity. In the latter case, longer response time was required for higher target velocity.

Acknowledgements - This study was made possible by a grant from the Electricite de France "Club Vision" No. 830143, Paris, and the Committee for Prevention and Research in Occupational Health at the Labor and Welfare Ministry, Jerusalem. The work of Thierry Vieville was supported by a grant from the Centre National d'Etudes Spatiales. The authors are indebted to Professor A. Fuchs for his comments.

\section{REFERENCES}

Barmark, N. H. (1970). Modification of eye movements by instantaneous changes in velocity of visual target. Vision Research, 10, 143!-1441.

Becker, W. \& Jürgens, R. (1979). An analysis of the saccadic system by means of double step stimuli. Vision Research, 19, 967-984.

Carpenter, R. H. S. (1977). Movements of the eyes. London: Pion.

Eckmiller, R. A. (1981). A model of the neural network controlling foveal pursuit eye movements. In Fuchs, A. \& Becker, W. (Eds.) Progress in oculomotor research. Amsterdam: Elsevier/North Holland.

Fuchs, A. (1971). The saccadic system. In Bach-y-Rita P., Collins, C. C. \& Hyde J. E. (Eds.) The control of eye movements. London: Academic Press.

Heywood, S. \& Churcher, J. (1981). Saccade to step-ramp stimuli. Vision Research, 21, 479-490.

Komoda, M. K., Festinger, L., Phillips, L. J., Duckworth, R. H. \& Young, R. A. (1973). Some observations concerning saccadic eye movements. Vision Research, 13, 1009-1020.

Logothetis, N., Fries, W. \& Popel, E. (1985). Extrafoveal pursuit as studied with the Rashbass paradigm. In McConkie, G. W. \& Menz, C. (Eds.) Eye movements and human information processing. Amsterdam: Elsevier/ North-Holland.

McKenzie, A. \& Lisberger, S. G. (1986). Properties of signals that determine the amplitude and direction of saccadic eye movements in monkeys. Journal of Neurophysiology, 56, 197-207. 
Rashbass, C. (1961). The relationship between saccadic and smooth tracking eye movements. Journal of Physiology, London, 159, 326-338.

Robinson, D. A. (1965). The mechanics of human smooth pursuit eye movement. Journal of Physiology, London, 174, 245-265.

Robinson, D. A. (1973). Models of the saccadic eye movement system. Kybernetic, 14, 63-70.

Tatsuoka, M. M. (1971). Multivariate analysis: techniques for educational and psychological research. New York: Wiley.
Wheeless, L. L., Boynton, R. M. \& Cohen, G. H. (1966). Eye movement responses to step and pulse-step stimuli. Journal of the Optical Society of America, 56, 956-960.

Wyatt, H. J. \& Pola, J. (1981). Slow eye movements to eccentric targets. Investigative Ophthalmology and Visual Science, 21, 477-483.

Zambarbieri, D., Schmid, R., Magenes, G. \& Prablanc, C. (1982). Saccadic responses evoked by presentation of visual and auditory targets. Experimental Brain Research, $47,417-427$. 